Artigos

Volume 9 - $2019 \mid$ n. 16

\title{
La Relación Fiscal-Educativa en Argentina: entre el centralismo de los recursos y el federalismo de los gastos
}

\author{
José Agustín Claus \\ Facultad Latinoamericana de Ciencias Sociales (FLACSO), Buenos Aires - Argentina
}

\section{Resumen}

En la última década, la historia educativa Argentina registró un inédito suceso, el crecimiento sostenido del financiamiento educativo por más de una década. No obstante, como sucede en las políticas educativas, se desperdició una coyuntura excepcional para impulsar las reformas estructurales de la educación. Este artículo aborda las características históricas del federalismo fiscal-educativo en el marco de una administración educativa enmarcada por la puja entre centralismo de los recursos (del Estado Nacional) y el federalismo de los gastos (en los Estados subnacionales) y el intento de superar esta puja mediante las políticas de recentralización del Estado nacional en materia de financiamiento educativo entre los años 2003 y 2015. Como conclusión, se detallan los principales hitos de la relación fiscal-educativa que propició la (excepcional) coyuntura y que la política educativa no supo (o no quiso) capitalizar como escenario para impulsar las reformas educativas del accionar estatal.

Palabras-clave: Financiamiento Educativo. Federalismo Fiscal. Descentralización. Centralización. Estado.

\section{The Fiscal-Educational Relationship in Argentina: between the centralism of resources and the federalism of expenses}

\begin{abstract}
In the last decade, Argentina's educational history registered an unprecedented event, the sustained growth of educational financing for over a decade. However, as in education policies, an exceptional situation was wasted to promote structural reforms in education. This article addresses the historical characteristics of fiscal-educational federalism within the framework of an educational administration framed by the provision between centralization of resources (of the National State) and federalism of expenditures (in subnational states) and the attempt to overcome this bidding by means of the recentralization policies of the national State in the matter of educational financing between 2003 and 2015. In conclusion, the major milestones of the fiscal-educational relationship that led to the (exceptional) situation and that the educational policy did not know (or did not want to) capitalize as a scenario to promote educational reforms of state action are detailed.
\end{abstract}

Palavras-chave: Educational Financing. Fiscal Federalism Decentralization. Centralization. State. 
La Relación Fiscal-Educativa en Argentina

\section{Introducción}

En los últimos años se registró un proceso inédito en la historia de la política educativa en Argentina, el crecimiento sostenido del financiamiento educativo ininterrumpido por más de una década. Aunque como, con frecuencia sucede en la educación del país, se desperdició una coyuntura excepcional para impulsar e implementar las reformas estructurales del sector que permitan imprimirle mayor equidad y justicia distributiva en el sistema educativo nacional.

Con el impulso de la Ley Nacional de Financiamiento Educativo, curiosa novedad institucional en educación, se procuró alcanzar el $6 \%$ del PIB como estrategia para superar la restricción presupuestaria históricamente preponderante en el sector educativo pudiendo correr como eje principal de discusión la escasez de recursos como escenario para la discusión e implementación de políticas educativas bajo el enfoque de derecho a la educación.

La evidencia empírica a lo largo de la historia del sistema educativo permitió identificar esta coyuntura como un hito de la relación fiscal-educativa en Argentina, sustentada a lo largo de décadas en un esquema de financiamiento basado en el centralismo de los recursos por parte del Estado Nacional y del federalismo de los gastos en los Estados subnacionales.

A lo largo del artículo, organizado en tres secciones, se desarrollan las principales características históricas de la relación fiscal-educativa en el marco del esquema de distribución de los recursos como marco fiscal de la administración educativa, el comportamiento y evolución del gasto sectorial enmarcado por la puja entre centralismo de los recursos y federalismo de los gastos y, por último, la política de recentralización del Estado nacional en el financiamiento educativo entre los años 2003 y 2015.

Como conclusión, se detallan los principales hitos identificados en el marco de la relación fiscal-educativa que propició la (excepcional) coyuntura y que los actores de la política educativa no supieron (o no quisieron) capitalizar como escenario de implementación de políticas educativas del accionar estatal, y como desafíos pendientes, algunos ejes que se podrían encomendar como propuesta de coordinación del cofinanciamiento entre el centralismo de los recursos y el federalismo de los gastos en el afán de imprimir mayor equidad y justicia distributiva en el sistema educativo nacional.

\section{El marco fiscal: el centralismo de los recursos}

El marco de las relaciones fiscales entre los niveles de gobierno se encuentra en permanente construcción desde la conformación del proyecto de Nación en Argentina. La puja histórica entre los distintos niveles de gobierno (Nación, Provincias y Ciudad de Buenos Aires) respecto de las responsabilidades de los recursos y los gastos, ha sido escenario constante de reforma y contrarreforma, en la cual, la educación no se encontró ajena.

La rama de las finanzas públicas preocupada por la comprensión del federalismo fiscal aborda los componentes principales de esta relación mediante dos perspectivas de análisis que establece, por un lado, la captación de los recursos y, por otro lado, la distribución de los gastos entre los niveles de gobierno. Desde la perspectiva horizontal, se diseña la distribución de responsabilidad de los tipos de finalidades y funciones que cada área de los niveles de gobierno se harán cargo y, desde la perspectiva vertical, se configura qué nivel de gobierno se responsabiliza de la provisión de los distintos bienes y servicios. Naturalmente, la determinación de las responsabilidades de los gastos por nivel de gobierno, se deberían 
La Relación Fiscal-Educativa en Argentina

asignación los niveles de recursos y esquemas de financiamiento para garantizar la sustentabilidad del esquema federal, por un lado, la perspectiva la distribución de responsabilidades y los recursos económicos según los distintos niveles de gobierno.

En esta línea, cuando se alude al federalismo fiscal, se remite al acuerdo de coordinación y conflicto entre los distintos niveles en relación con las decisiones de financiamiento y gastos del Estado. Asimismo, resulta clave que esta relación permita (o al menos) garantizar el equilibrio fiscal horizontal (en el interior de cada nivel de gobierno), como en la relación vertical (entre niveles distintos) mediante el sistema de transferencias de recursos entre el estado nacional y los subnacionales (ACCOTO; MARTíNEZ; MANGAS; PAPARAS, 2016).

Buena parte de los estudios y especialistas que se dedicaron al estudio del federalismo fiscal en Argentina, entre los cuales se pueden destacar a Bravo (1968), Jimenez (2002; 2004), Cetrángolo (2002; 2004), Piffano (2005), Porto (2003; 2008) y Morduchowicz (2009; 2010), (entre otros) y aunque cada uno de ellos tiene postulados diferentes para la comprensión de las causas del esquema federalismo fiscal en Argentina, la mayoría argumentan un consenso respecto de la identificación como él nudo crítico del origen del esquema federal, la falta de un sistema federal de distribución de recursos sustentado en criterios objetivos que permita cumplir con criterios devolutivos y distributivos que genere sustentabilidad en las finanzas del gobierno nacional y de las jurisdicciones provinciales.

En este sentido, la tensión existente entre centralización y descentralización remite al problema de la equidad y de la distribución de poder en el sistema educativo, y esto de alguna manera es lo que determina las iniciativas en una u otra direccionalidad (ALMANDOZ, 2002).

Desde mediados del Siglo XX la relación fiscal entre el Estado Nacional y los Estados provinciales estuvo determinada por una significativa brecha vertical que se amplío con el transcurso del tiempo, en la medida que el gobierno nacional incrementó de forma paulatina pero constante, la potestad de las principales fuentes recaudatorias.

En el gráfico 1 siguiente, se expone el comportamiento de los recursos y los gastos entre 1961 y 2013, con el objeto de mostrar cómo fue su evolución en la cual se destaca el centralismo de los recursos por parte del estado nacional y de la federalización de los gastos a cargo de los estados provinciales principalmente y en menor medida de los municipales. 
La Relación Fiscal-Educativa en Argentina

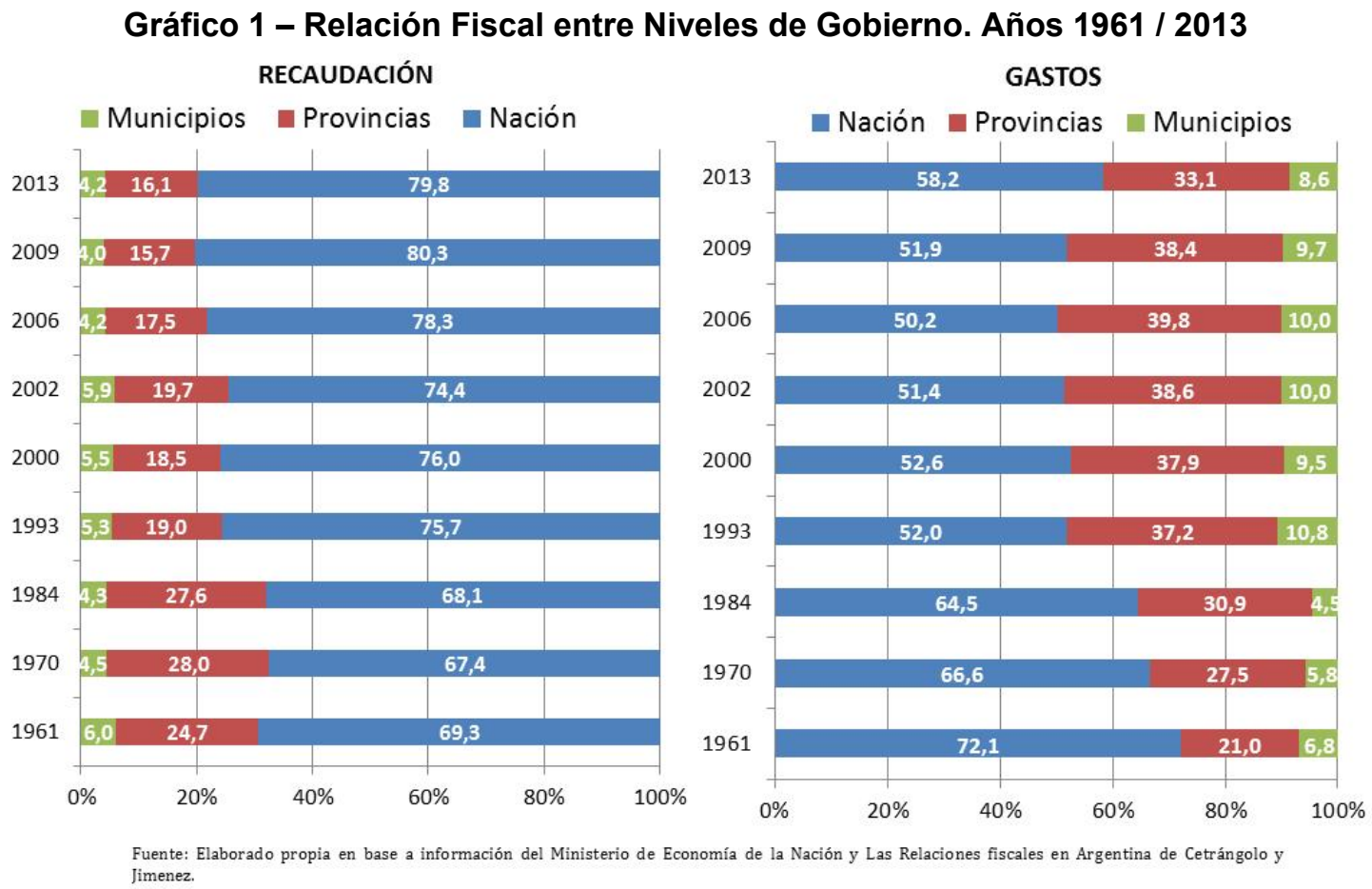

Como se muestra, la evolución de la participación de la Nación en la recaudación se incrementó de forma significativa a la vez que disminuyó de manesa sostenida su responsabilidad en los gastos, y contrariamente, las provincias redujeron su apropiación en el total de los recursos e incrementaron su participación en el total de los gastos públicos consolidados. Este marco institucional de escaza delimitación de las funciones y responsabilidad de cada nivel de gobierno generó distintos procesos de reasignación de funciones a lo largo de la historia del federalismo fiscal y constituyó el escenario de descentralización de los servicios educativos de la educación no universitaria en Argentina, al menos en el financiamiento educativo.

Desde los años de la década del sesenta hasta la última dictadura cívico-militar del año $76^{\circ}$, la participación de los gastos a cargo del Estado nacional, se redujo de manera progresiva (en el aparatado siguiente se detalla el sector educativo) hasta que en el año 1976 se descentralizaron los servicios educativos de educación primaria, la cual había sido la principal política de expansión a cargo del gobierno nacional impulsado fuertemente por la Ley Laínez¹.

Esta primera etapa de descentralización, junto con otros servicios como salud, generó que la Nación reduzca en casi diez puntos porcentuales su participación en la distribución de los gastos públicos consolidados. Desde los años de la apertura democrática, la tendencia a la baja de su participación en los gastos continuó creciendo, pero no fue hasta la segunda etapa de descentralización en el año 1992 donde se profundizó y culminó el proceso de descentralización comenzado con la dictadura cívico-militar.

1 Resulta muy interesante la dinámica incorporada por la Ley sancionada a comienzos del siglo XX por la cual, el Senador Laínez, de allí su nominalización a la Ley, estableció la posibilidad que el estado nacional construya y amplié la cobertura de la educación primaria, potestad provincial, pero de magro cumplimiento constitucional. Para ampliar se recomienda la lectura de la publicación "A 100 años de la Ley Laínez" del Ministerio de Educación de la Nación. 
Resulta notorio que no fue hasta la recuperación democrática y en el año $1988^{2}$ con vigencia del actual régimen de Coparticipación Federal de Impuestos, con carácter provisorio de vigencia de dos años y hasta que se sancioné y promulgue una nueva ley que la reemplace. No obstante, no se sancioné ninguna ley reemplazante y sólo se mantuvo el esquema de repartición de recursos sancionado.

El esquema de distribución estipulado en la Ley № 20.221 procuró subsanar la discusión existente entre los años de la recuperación democrática generada por la inexistencia de un marco regulatorio en el reparto de los recursos a nivel nacional. En efecto, la fórmula establecida constituyó en una distribución del $56,6 \%$ para las provincias, $42,3 \%$ para la Nación y el $1 \%$ para la constitución de un fondo de las transferencias discrecionales del estado nacional, como mecanismo de generar asistencia en situaciones contracíclicas o de crisis económicas, recurrentes en los estados provinciales. Luego de esta distribución denominada primaria, entre los niveles de gobierno Nación y provincias, se definió una distribución secundaria mediante la cual se estableció la participación relativa de cada una de las jurisdicciones provinciales existentes en ese momento histórico.

Hace más de cincuenta años, Bravo elaboró un conjunto de principios para abordar el régimen fiscal de la educación nacional en Argentina y éstos en la actualidad cumplen cincuenta años, pero su carácter de comprensión e interpretación resultan más que actuales, modernos.

Estos principios reflejan el esquema de financiamiento educativo nacional, sustentados en la existencia de cuatro 'Is', a) Inorganicidad, cuyas afectaciones especifican surgen y se implementan producto de la coyuntura y no forman parte de una planificación sistematizada, b) Inestabilidad, mediante la sanción de leyes que no llegan a implementarse o al comienzo de su implementación se discontinúan, c) Incumplimiento, no se especifican objetivos de política a los cuales se destinaran los recursos incrementales y la d) Insuficiencia, generada por un lado, por la restricción presupuestaria, pero por otro, debido a la falta de planificación y programación de los gastos que genera imprecisión respecto de las necesidades y por ende limita la posibilidad de conocer con precisión la suficiencia y/o magnitud de las necesidades de financiamiento (BRAVO, 1968).

En la Argentina, constitucionalmente es potestad de las jurisdicciones provinciales la expansión de nivel de educación primaria, razón por la cual, el Estado Nacional, no debía destinar recursos para su financiamiento. Contrariamente, producto de la génesis y configuración del sistema educativo nacional, fue el Estado Nacional, quién impulsó a través de políticas de recentralización financiera la expansión del nivel primario, como así, generó el impulso para la conformación de un sistema educativo nacional.

2 En 1973 se promulgó la Ley № 20.221 que estableció un mecanismo de régimen de coparticipación de recursos, derogada en 1976 con la dictadura cívico-militar. 


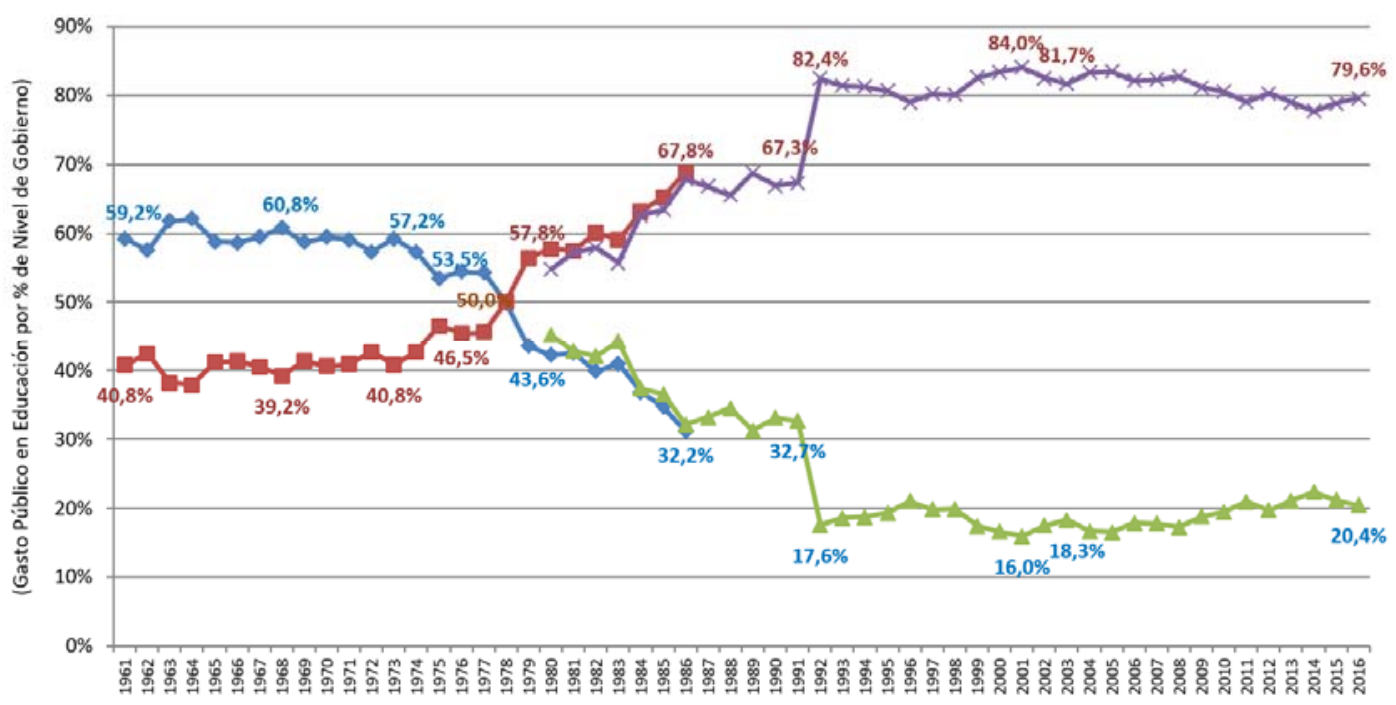

Puente: Elaboración propia en base a información Dirección de Gasto Social Consolidado/MECON e INDEC del Ministerio de Hacienda de la Nación. Periodo 1980-1989: considerado de El gasto público en educaclón de Humberto Petrel. Perlodo 1991-2000: considerado de el Gasto Público Educativo 1991-2000/PCSE y Perlodo 2001-2015: CGECSE/Ministerio de Educaclón de la Nación. Año 2016: elaboración
propia y cierre provisorio elaborado a julio/18. Serie de PIB: serie empalmada considerando base 1993 para aplicar a base 2014.

Como se expone en el gráfico 2, la segunda etapa de descentralización comenzada desde el año 1991 y consagrada en 1992 con la Ley Federal de Educación, impulso la década denominada de la Reforma Educativa. Pese a las discrepancias de distintos especialistas, dedicados a estudiar la multiplicidad de variables intervinientes durante este periodo, a los fines de nuestro objeto de análisis se destacan dos hitos.

En primer lugar, el Estado Nacional, en un contexto de reforma estructural del aparato del Estado, culminó el proceso de descentralización de los servicios de educación media (actual secundaria) y de los institutos de formación docente, manteniendo sólo las instituciones de educación superior universitaria. En efecto, en el cuerpo de la Ley Federal de Educación del país con casi doscientos años de historia, aunque con más dictaduras que gobiernos democráticos, se estipuló la meta de alcanzar el $6 \%$ del PIB.

Esta meta procuró capturar mayores recursos en el marco de expansión de la cobertura educativa obligatoria en función de la nueva estructura del sistema, la profesionalización de los docentes, las políticas compensatorias y la mejora de la calidad educativa.

En segundo lugar, durante la década del noventa, desde 1990 hasta la 1994, se cumplieron de forma parcial los niveles de recursos incrementales por nivel de gobierno, en efecto la Ley de Transferencia del año 1992, estableció el mecanismo de asignación de recursos que en el año 1995 se vio restringido incidido producto de la crisis internacional del Tequila que impactó en la reducción del presupuesto destinado para el año subsiguiente restableciendo la persistente crisis del financiamiento educativo nacional.

Luego de la crisis del año 2001 y con el recambio de época desde el año 2003 se reposicionó al Estado Nacional como actor principal para la búsqueda de mayor equidad y justicia en el sistema educativo. En el año 2005, el financiamiento educativo se posicionó en la agenda de política pública mediante la sanción de la Ley Nacional de Financiamiento Educativo, que introdujo un mecanismo que afectaba recursos para sostener el incremento 
estipulado, con un conjunto de objetivos y metas de políticas educativas, y sobre todo, la ley dispuso una contribución relativa del incremento del gasto por parte del gobierno nacional mayor al existente en el momento de promulgación de dicha Ley.

La Ley Nacional de Financiamiento Educativo, que detallamos en los apartados siguientes, consagró de manera inédita, desde la década del sesenta y al menos desde la apertura democrática, el rol del Estado Nacional como vector y garante del financiamiento educativo, recentralizando políticas educativas, que históricamente descentralizó, pero que mediante el impulso de éste esquema de financiamiento procuró reposicionar al Estado Nacional como actor fundamental en el sistema educativo federal y descentralizado con persistencia de grandes desigualdades en su dispersión territorial.

\section{La responsabilidad educativa: el federalismo de los gastos}

Durante la década del ochenta, la educación en Argentina no estuvo ajena a los ciclos y vaivenes macroeconómicos, en síntesis, en tiempos de crecimiento más recursos, en tiempos de recesión, menos recursos. No obstante, la economía durante la década del ochenta prácticamente estuvo subsumida en una crisis continua, y como reflejo de ello, durante prácticamente todos los años de la misma, el financiamiento educativo oscilo en torno al $2 \%-3 \%$ del PIB.

En los inicios del Plan de Convertibilidad, proceso inédito en la historia económica Argentina, se ambicionó a generar un crecimiento sostenido del financiamiento educativo con el objetivo de alcanzar la meta del $6 \%$ del PIB en el plazo de cinco años o duplicar los niveles de gasto sectorial respecto del registrado en 1992 con la sanción de la Ley Federal de Educación. El compromiso consistió en incrementar el presupuesto a una tasa del $20 \%$ interanual instrumentando acuerdos bilaterales entre la Nación y las Provincias como medio de asignación de los recursos en el marco del Pacto Federal Educativo, firmado en el año 1994.

Mediante dicho compromiso, la Ley no estableció, por un lado, reglas objetivas para la distribución y asignación de los recursos a las jurisdicciones provinciales, razón por la cual, primó la discrecionalidad y el acuerdo político como medio de establecimiento de la suficiencia de la magnitud a financiar en cada una de las jurisdicciones provinciales.

Por otro lado, dicha ley no estableció mecanismos para la captura de los fondos que permitieran sostener el incremento sostenido de los recursos, ya que la vía había sido presupuestaria, sin afectación específica, como todo presupuesto que opera en función de la coyuntura económica y política de gobierno, no sobrevivió al proceso de ajuste estructural que derivó los últimos años de dicha década.

En el gráfico siguiente se destaca el comportamiento de la inversión, en particular de la década del noventa, en el cual se partió del 2,9\% del PIB en el año 1990, alcanzando el máximo en el año 2001 en el 4,5\%, incrementándose en sólo 1 punto porcentual del PIB, cuando se aspiraba a duplicar el nivel de inversión comprometida por la Ley Federal de Educación.

Respecto al comportamiento de los gastos, la relación fiscal-educativa, entre la nación y las provincias, claramente se destaca que durante la década del ochenta la Nación redujo 
La Relación Fiscal-Educativa en Argentina

de forma sistemática su participación en el gasto, mientras que las jurisdicciones provinciales registraron niveles de crecimiento continuo y significativo bajo el mismo periodo.

\section{Gráfico 3 - Evolución del Gasto Público Consolidado en Educación, por Nivel de Gobierno y en \% del PIB. Años 1980 / 2016}

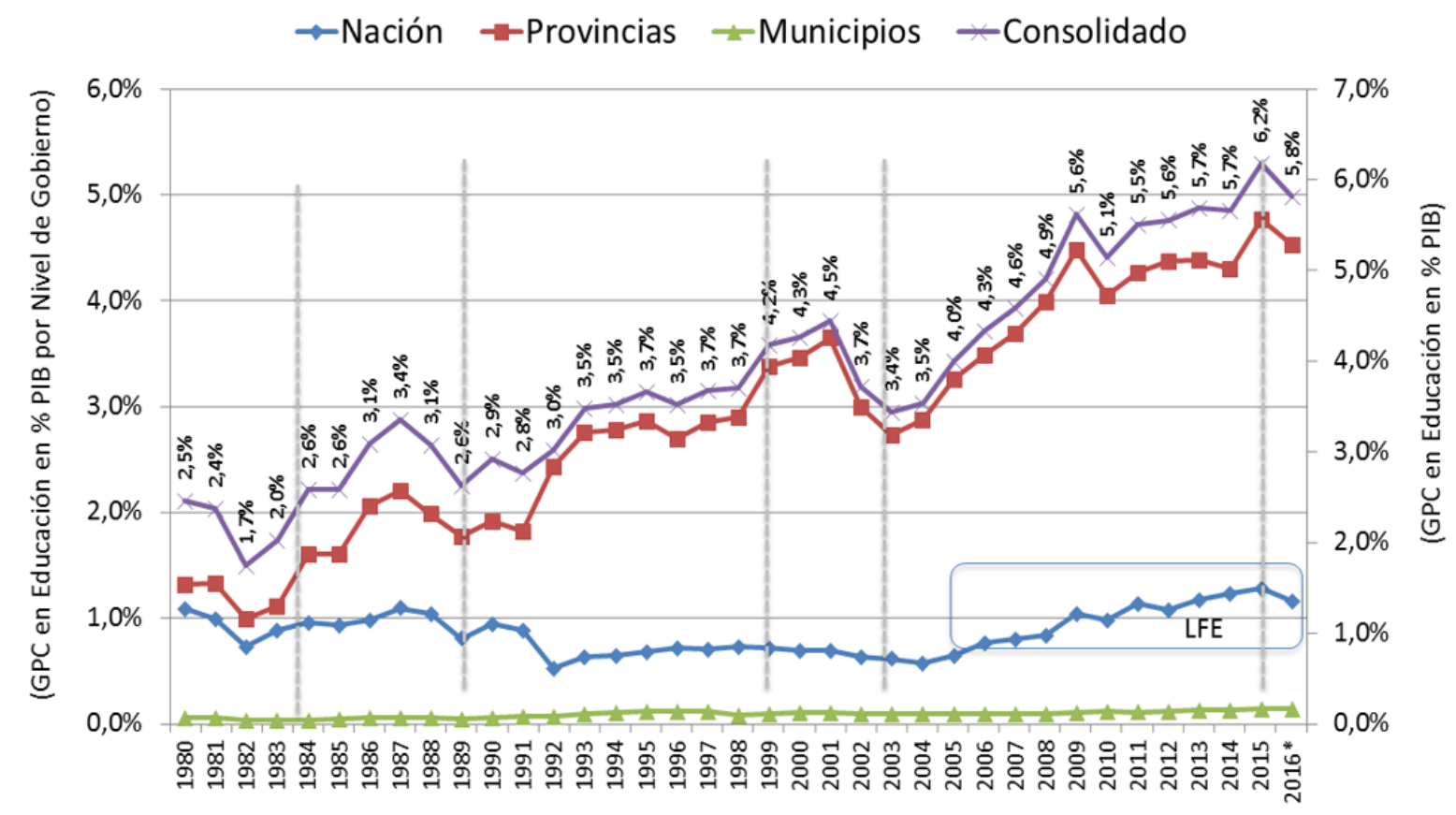

Fuente: Elaboración propia en base a información Dirección de Gasto Social Consolidado/MECONe INDEC del Ministerio de Hacienda de la Nación, Periodo 1980-1989: considerado de El gasto público en educación de Humberto Petrei. Periodo 1991-2000: considerado de el Gasto Público Educativo 1991-2000/PCSE y Periodo 2001-20
propia y cierre provisorio elaborado a julio/18. Serie de PIB: serie empalmada considerando base 1993 para aplicar a base 2014.

Uno de los principales impactos registrados producto de la transferencia y desentendimiento por parte del Estado Nacional mediante la culminación de la descentralización de los servicios educativos, radicó en los salarios docentes provincializados, ya que éste se constituyó como el principal rubro de gasto en la inversión educativa consolidada.

En sintonía, Cetrángolo y Gatto, plantearon que el proceso de descentralización dio una total libertad de acción a cada gobierno para la fijación de los niveles salariales. La divergencia en los niveles salariales provinciales -que ya existía- pareció haberse profundizado al momento de la transferencia de las escuelas primarias y secundarias nacionales.

De hecho, la política seguida por cada provincia no siempre correspondió con sus posibilidades financieras, ya que algunas jurisdicciones provinciales con menores recursos genuinos llevaron a la práctica políticas salariales docentes insostenibles de forma autónoma y debieron apelar al apoyo del Tesoro Nacional. En cada caso o circunstancia, la política salarial ha sido el resultado, entre otros factores, del diferente grado de responsabilidad fiscal de cada gobierno, de su poder de negociación con el gobierno nacional y de su relación con los sectores sindicales docentes.

Lo cierto es que habida cuenta de la ausencia de coordinaciones específicas y de conflictos permanentes en la relación fiscal Nación-provincias, la descentralización educativa terminó jugando como una pieza subordinada del problema más general. La educación se convirtió en un canal adicional para obtener directa o indirectamente apoyo financiero extraordinario del gobierno nacional (CETRÁNGOLO; GATTO, 2002). 
La llegada del colapso del Régimen de Convertibilidad en los años 2001 y 2002 implicó una aguda contracción en términos reales de la inversión consolidada que se extendería hasta el año 2003. En efecto, el nivel de gasto consolidado de dicho año fue similar al de doce años previos, retrotrayendo el nivel a los años iniciales de la década del noventa. Desde el año 2003, se observaron algunos signos de recuperación de la actividad económica y se comenzó a transitar un proceso de crecimiento económico con tasas reales significativas.

En este marco, desde el año 2004, se observó un crecimiento de la inversión consolidada en educación, ciencia y tecnología conjunta de la Nación y las provincias, a la vez, que el nivel del PIB se expandió a tasas muy significativas.

Claus afirma que no fue hasta el año 2005 que se sancionó la Ley de Financiamiento Educativo $\mathrm{N}^{\circ} 26.075$, con vigencia a partir del año 2006, con la cual se introdujeron mecanismos de asignación de recursos al sistema educativo de manera progresiva y constante. Esta norma implicó una novedad en términos de las herramientas fiscales, mediante la introducción de reglas claras para ambos niveles de Gobierno, nacional y Provincial, proponiéndose alcanzar el $6,0 \%$ del PIB destinado a la educación, ciencia y tecnología en el año $2010^{3}$.

En dicho marco, la Ley promovió la distribución de responsabilidades de financiamiento para ambos niveles de gobierno, con la proyección de metas anuales de inversión para el período 2006-2010. En la cual, se comprometió, que el gobierno nacional contribuya con el $40 \%$ y los gobiernos provinciales con el $60 \%$ de los recursos incrementales estipulados para alcanzar las metas de inversión (CLAUS, 2011).

En este periodo, impulsado entre los años 2003 y 2005, por políticas de ingresos generales y en particular en los salarios docentes, e institucionalizada por la Ley desde el año 2006 se generó un inédito y sostenido crecimiento real de la inversión educativa elevándola del histórico 3\% del PIB de las décadas ochenta y noventa, hasta alcanzar en el 2015 el 6\% del PIB.

Lo inédito de dicho proceso de financiamiento educativo, es que mientras la Nación duplicó su participación del PIB, elevándose del histórico $0,6 \%$ al inédito $1,2 \%$, las provincias incrementaron su participación en un $66 \%$, elevando su nivel de gasto del $2,8 \%$ al $4,8 \%$ en el nivel máximo del año 2015.

El análisis de la descentralización del gasto se presenta en el siguiente gráfico, en el cual, se evidencian los cambios de participación del financiamiento educativo en el marco de la relación fiscal-educativa por nivel de gobierno.

Durante la década del $80^{\circ}$, el Estado Nacional, comenzó cofinanciando con más del $40 \%$, las provincias con más del $55 \%$ y el resto a cargo de los municipios con cerca del $2 \%$.

3 En el momento de diseño y formulación de la implementarse la Ley de Financiamiento Educativo, se encontraba vigente el PIB calculado con base en el año 1993. Las metas de inversión se establecieron sobre la base de proyecciones de este PIB para los años 2005 a 2010. Sobre esta base, la meta de inversión en educación, ciencia y tecnología, puede decirse que la meta de inversión se cumplió en el año 2010. No obstante, el recálculo del PIB publicado en 2014 (y revisado en 2016) con base en el año 2004 elevó en términos nominales el valor contra el que se compara el gasto en educación. Tomando como punto de partida los valores recalculados, la Ley habría partido de un gasto en Educación, Ciencia y Tecnología de 4,2\% en 2005 (en lugar de $4,6 \%$ ) y habría llegado en 2010 a 5,4\%. Con estos valores actualizados, puede decirse que la meta de inversión en Educación, Ciencia y Tecnología recién fue cumplida en 2013, mientras que la meta de inversión únicamente en Educación fue alcanzada en el año 2015. 
Estas relaciones producto de la descentralización entre 1991 y 1992, se vieron notoriamente modificadas, en la cual la Nación, registró una reducción de 14 puntos porcentuales y las provincias incrementaron su participación en 15 puntos porcentuales.

\section{Gráfico 4 - Relación del Gasto Educativo por Nivel de Gobierno: Nación, Provincias y Municipios. Años 1980 / 2016}

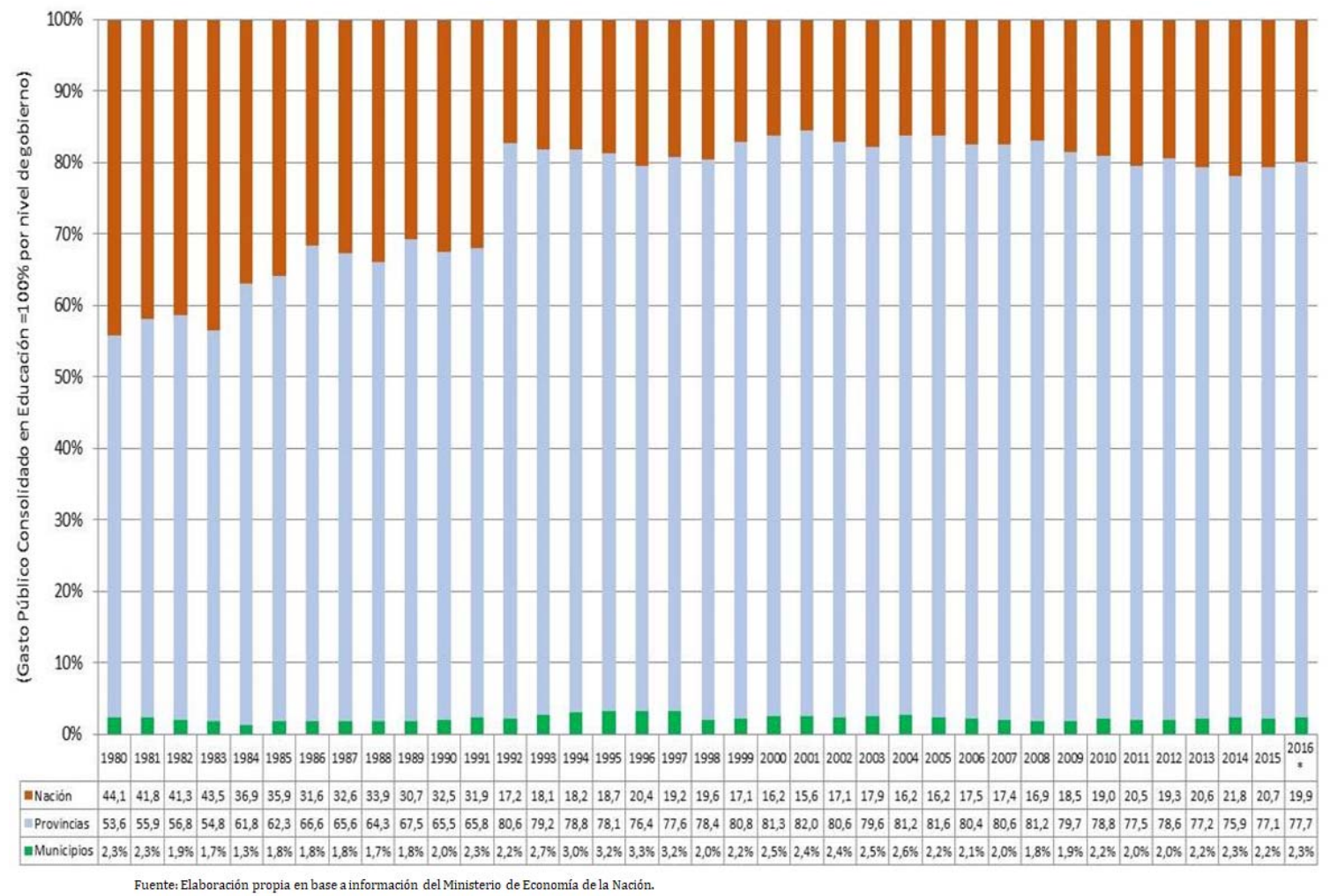

Durante los años 2003 - 2015, la participación del Estado Nacional, registró un crecimiento leve, pero continuo, incrementándose en dos puntos porcentuales que se vieron reducidos por parte de las provincias, evidenciando un significativo esfuerzo adicional respecto del incremento consolidado, ya que de ese aumento, la nación se esforzó más en términos relativos que el conjunto de las jurisdicciones provinciales.

La responsabilidad en el cofinanciamiento de la educación resulta intrínseca a la relación fiscal-educativa que el sistema educativo nacional configuró a lo largo de los jóvenes treinta y cinco años de democracia, aún marcados e influidos por parte de las reformas de la última dictadura cívico-militar y las reformas de los años noventa. Queda pendiente entre los preocupados por lo educativo, consensuar y establecer reglas de coordinación federal respecto de la responsabilidad y obligación del rol del Estado Nacional en el marco del federalismo fiscal y educativo.

\section{La recentralización del Estado Nacional}

Como consecuencia del proceso de transferencia y reforma del sistema educativo en Argentina sin la consecuente distribución de recursos que permitan sustentar dicha descentralización de los servicios, se generaron distintos hitos que obligaron al Estado Nacional a comenzar un proceso de recentralización de las políticas educativas. 
Caben dos contextos políticos-económicos bien diferenciados, que permiten indagar la recentralización del rol del Estado Nacional. En primer lugar, por necesidad y gobierno del sistema ante los reclamos durante los años noventa, y en segundo lugar, por convicción y proyecto de Estado-Educación durante los años del periodo de la ley de financiamiento educativo.

El primer contexto político-económico remite al problema derivado por la restricción presupuestaria que permita encauzar el problema de lo salarios docentes, en el cual se realizó una propuesta de docentes a comienzos del año 1997 con la instalación de la Carpa Blanca, como reclamo de mejora de los salarios docentes y condiciones laborales.

En efecto, en 1998 el Ministerio de Educación presentó una propuesta legislativa para la creación de un nuevo impuesto sobre los automóviles, generando un fondo de financiamiento específico para financiar mejoras salariales docentes. Este proyecto fue aprobado e implementado durante el primer año, aunque con un escazo nivel de recaudación respecto de la meta propuesta que limito y licuo el impacto de jerarquización del salario docente.

En dicho marco, y producto de las promesas electorales, en el cual el gobierno releecto se comprometió a financiar vía presupuestaria, nuevamente presupuesto, la conformación de un Fondo Nacional de Salario Docente.

Este nuevo instrumento de financiamiento educativo se denominó Fondo Nacional de Incentivo Docente, mediante el cual el Estado Nacional se comprometió a cofinanciar los salarios docentes, descentralizados, excepto los Universitarios, en todas las jurisdicciones del país.

La principal ventaja de dicho fondo consistió en su faceta instrumental, debido a que consistió en una suma fija por cargo, sin ningún tipo de consideración respecto al ingreso de las personas docentes, antigüedad, titulo, ámbito o localización de las instituciones en las cuales se desempeñan.

Como desventaja, en el marco de la relación fiscal-educativa, la incorporación de dicho FONID significó incentivos o señales contradictorias a las jurisdicciones provinciales, debido a que, por un lado, el estado nacional se posicionó como co-financiador de los salarios docentes, y por las características de dicho fondo, no se incentivó el esfuerzo, la capacidad o la eficiencia en el uso de los recursos limitados por parte de las jurisdicciones provinciales para incrementar los niveles salariales supeditados a la definición del aporte nacional.

Entre las políticas de ingresos del estado nacional, en el periodo 2004 a 2015, en el sector educativo se consagró la Paritaria Nacional Docente, desde el año 2008, en la cual, por primera vez en la historia educativa se estipuló en acuerdo federal el salario mínimo docente garantizado a nivel nacional.

En este marco, la institucionalización de un espacio con representación federal, en la cual el Gobierno Nacional y Provinciales se reúnen con los representantes gremiales de carácter nacional, estableciendo no sólo el salario mínimo sino un conjunto de condiciones que hacen a la capacitación, carrera docente y condiciones laborales.

Tal como analizó Claus, el impacto de la paritaria nacional docente, mediante la estipulación de salario mínimo a nivel nacional permitió reducir la brecha de la desigualdad 
La Relación Fiscal-Educativa en Argentina

docente en las provincias en 22 puntos porcentuales, a la vez que se estableció el Programa Nacional de Compensación Salarial Docente (CLAUS, 2017).

No obstante, resulta interesante, el destino de la política de recentralización del gasto a cargo del Estado Nacional, en función de las instituciones bajo su dependencia. Es decir, durante la década del noventa, el gasto preponderante se concentró en la Educación Universitaria y Ciencia y Tecnología.

De forma contraria a lo sucedido en los años noventa, desde el 2003, se observa un pico máximo del $35 \%$ del gasto educativo nacional destinado a la Educación Básica (Inicial, Primaria, Secundaria y modalidades) con el objeto de recentralizar las políticas nacionales en materia educativa.

Gráfico 5 - Distribución del Gasto del Ministerio de Educación de la Nación por Nivel Educativo. Años 1993 / 2014

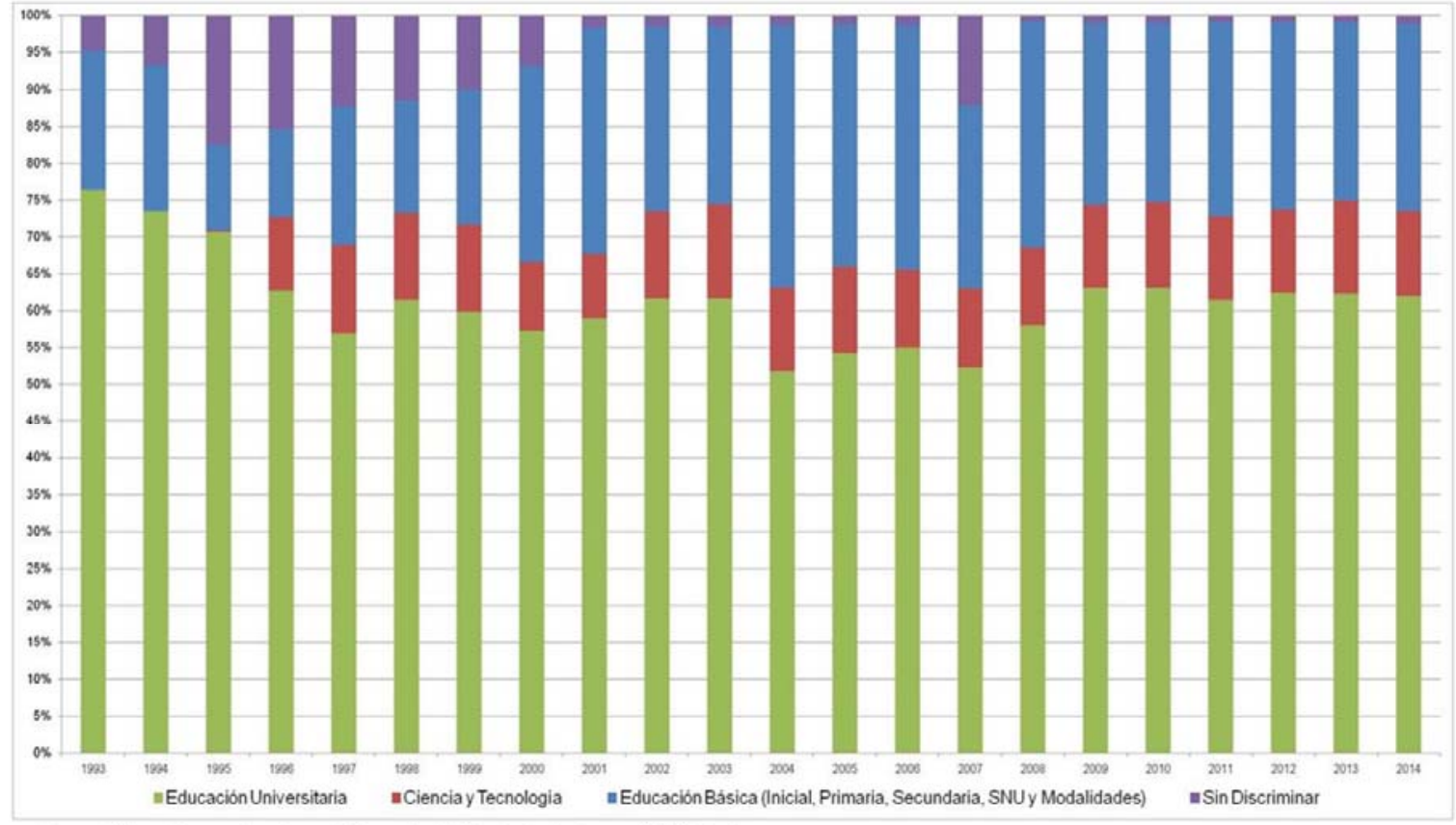

Fuente: Elaboración propia en base a información del Ministerio de Economía de la Nación.

La situación compleja del esquema federal aún permanece irresuelta respecto del modelo del financiamiento educativo y de las políticas educativas enmarcadas en la relación fiscal-educativa del Gobierno Nacional y de las jurisdicciones provinciales.

Es decir, es mediante la vía presupuestaria que se procura mejorar la equidad del gasto educativo en las distintas provincias o bien se requiere incorporar nuevas reglas de coordinación fiscal-educativa mediante las cuales el estado nacional institucionalice su rol consagrado en la Ley Nacional de Educación mediante un esquema de financiamiento sustentable en el mediano y largo plazo.

En la misma línea queda pendiente de resolución del rol del estado nacional en el cofinanciamiento salarial de los docentes de las jurisdicciones provinciales, para lo cual, habría que repensar el esquema de asignación de dichos aportes en función de considerar la capacidad recaudatoria, la necesidad de cofinanciamiento y el esfuerzo realizado por las jurisdicciones provinciales. 
La Relación Fiscal-Educativa en Argentina

\section{Conclusión}

El histórico reclamo por incrementar el financiamiento educativo no obedece a una apelación políticamente correcta sino a la necesidad de generar algunas de las condiciones mínimas y consensuadas para garantizar una educación de equidad y calidad para toda la sociedad.

Resulta reiterativo, pero no menor, que la Ley de Financiamiento Educativo consistió en un mero instrumento y, no un objetivo en sí mismo, a partir del cual se esperaba la voluntad política para impulsar los cambios necesarios en término de los principales desafíos aún pendientes. No obstante, resultó evidente el significativo e inédito avance respecto de la situación preexistente, y pese a todo no resulto poca cosa. En síntesis, el objetivo de la Ley se cumplió porque procuró incrementar en educación, ciencia y tecnología dos puntos porcentuales del PIB en el periodo de cinco años, independientemente de la metodología de medición como analizamos.

En este contexto, la educación de Argentina protagonizó de una situación positivamente inédita de disponibilidad de recursos para el sector, que correría del eje de la discusión, los resultados (pobres) del desempeño escolar, al menos como causa de la restricción de recurso, sino más bien en esta situación corresponde asumir la responsabilidad por el magro impacto a las acciones y efectividad de la política educativa adoptada.

De manera retrospectiva, el incrementó traccionado por dicha Ley, consistió en la recuperación real de los salarios docentes, históricamente deteriorados y para financiar la ampliación de los planteles y equipos docentes, aun creciendo a tasas superiores a la matrícula.

En este marco, la recuperación salarial acompaño el ciclo económico, la introducción de una afectación específica destinada a la educación en el régimen de Coparticipación Federal de Impuestos, registró un incremento significativo al inicio, pero luego se tornó laxa y no permitió a modificar la estructura del financiamiento educativo.

Resulta evidente que el enfoque de gasto consolidado como herramienta de financiamiento posee limitaciones significativas, primero, asociadas a la coyuntura y la fluctuación de la dinámica macroeconómica y, segundo, vinculadas con la política del gobierno mediante la cual, ante la falta de consenso como política de Estado, la puja entre descentralización y centralización de políticas fiscales-educativas permanecerá constante en el corto y mediano plazo.

Incorporar la perspectiva del largo plazo en el esquema del financiamiento educativo no puede esperar ante la posibilidad y voluntad política de mejorar el desempeño del sistema educativo, en base a un conjunto de reglas objetivas y un mecanismo de coordinación entre los niveles de gobierno de la educación que identifique responsabilidades por objetivos y acompañe con la asignación de recursos con plena transparencia de su trazabilidad y el Accountabily en el financiamiento educativo.

Como resulta en las políticas educativas, con buenas intenciones e incluso acciones necesarias y hasta imprescindibles, no resultan ser suficientes, se requiere incorporar la dimensión de racionalidad a los incrementos presupuestarios del financiamiento educativo. 
En síntesis, la lógica subyacente del financiamiento en el sistema educativo nacional ha permanecido invariable a lo largo de los últimos 35 años y aún no se plantea la necesidad de impulsar modificaciones estructurales en la búsqueda de mayor efectividad de la inversión.

Situación contraria, permanece con la determinación de cuál es el nivel de inversión educativa, a la que de distintos actores, exigen nuevos aumentos y aunque se aspire a elevar e incrementar la meta de inversión consolidada al 7\%, 8\% y/o 10\% del PIB en los próximos años, lamentaría augurar en base al análisis de la evidencia empírica, que si no se modifica el esquema subyacente estaremos con los mismos problemas estructurales irresueltos, pero eso sí, con un mayor nivel de inversión educativa inédito en Argentina que se necesita sostener en el mediano y largo plazo.

\section{Bibliografía}

ACCOTO, Alejandro López; MARTÍNEZ, Carlos R.; MANGAS, Martín; PAPARAS, Ricardo. Finanzas públicas y política fiscal: conceptos e interpretaciones desde una visión argentina. Buenos Aires: Universidad Nacional de General Sarmiento, 2016.

ALMANDOZ, María Rosa. Sistema Educativo Argentino: Escenarios y Políticas. Buenos Aires: Santillana, 2000.

BRAVO, Héctor. Régimen fiscal de la educación nacional. Documento de Trabajo. Cuadernos del Centro de Investigaciones Educativas (CICE). Buenos Aires, 1968.

CETRÁNGOLO, Oscar; GATTO, Francisco. Descentralización Fiscal en Argentina: restricciones impuestas por un proceso mal orientado. In: SEMINARIO "DESARROLLO LOCAL Y REGIONAL: HACIA LA CONSTRUCCIÓN DE TERRITORIOS COMPETITIVOS E INNOVADORES", 2002, Quito. Trabajo... Instituto Latinoamericano y del Caribe de Planificación Económica y Social (ILPES) de la Comisión Económica para América Latina y el Caribe (CEPAL), Quito, 2002.

CETRÁNGOLO, Oscar; JIMÉNEZ, Juan Pablo. Las relaciones entre niveles de gobierno en Argentina. Raíces históricas, instituciones y conflictos personales. Instituto Latinoamericano y del Caribe de Planificación Económica y Social (ILPES) de la Comisión Económica para América Latina y el Caribe (CEPAL). Serie N 47, Gestión Pública. 2004.

CLAUS, Agustín. El Financiamiento Educativo y las Condiciones Laborales de los Docentes. In: CONCURSO BICENTENARIO DE LA PATRIA, 2., 2011, Buenos Aires, Ministerio de Trabajo de la provincia de Buenos Aires. Buenos Aires, 2011.

CLAUS, Agustín. La Paritaria Nacional Docente y la política de Salarios Mínimos Docentes en la Argentina entre 2005 y 2016: Impacto, limitaciones y desafíos. Revista Estado y Políticas Educativas, Buenos Aires, Facultad Latinoamericana de Ciencias Sociales (FLACSO), Argentina, n. 9, 2017.

MORDUCHOWICZ, Alejandro; ARANGO, Aída. Diseño institucional y articulación del federalismo educativo. Instituto Internacional de Planeamiento Educativo de la Organización de las Naciones Unidas para la Educación, la Ciencia y la Cultura, 2010.

PIFFANO, Horacio. Notas sobre federalismo fiscal. Enfoques normativo y positivo. PrEBi/SeDiCl, Universidad Nacional de La Plata, Argentina, 2005. 
La Relación Fiscal-Educativa en Argentina

PORTO, Alberto. Capacidad, Necesidad y Situaciones Fiscales Provinciales en las Políticas Públicas Argentinas. Universidad Nacional de La Plata, Facultad de Ciencias Económicas. Documento sobre Federalismo Fiscal n. 6, 2003.

José Agustín Claus es Maestrando en Gestión Pública y Desarrollo Gubernamental y Licenciado en Administración por la Facultad de Ciencias Económicas de la Universidad de Buenos Aires (UBA). Docente de postgrado del Seminario de Economía de la Educación en la Maestría de Ciencias Sociales con Orientación en Educación de la FLACSO. Es investigador asociado a la Red Latinoamericana de Estudios sobre Trabajo Docente Argentina (ESTRADO) y al Programa Educación, Conocimiento y Sociedad del Área de Educación de la Facultad Latinoamericana de Ciencias Sociales (FLACSO).

ORCID: https://orcid.org/0000-0003-1834-3602

E-mail: agustinclaus@gmail.com

Recibido en 12 marzo 2019

Aprobado en 18 abril 2019 


\section{Editores do volume 9}

José Marcelino de Rezende Pinto - Universidade de São Paulo, São Paulo/SP, Brasil

Nalú Farenzena - Universidade Federal do Rio Grande do Sul, Porto Alegre/RS, Brasil

\section{Comitê Editorial}

José Marcelino de Rezende Pinto - Universidade de São Paulo, Brasil

Juca Gil - Universidade Federal do Rio Grande do Sul, Brasil

Theresa Adrião - Universidade Estadual de Campinas, Brasil Ângelo

Ricardo de Souza - Universidade Federal do Paraná, Brasil

Márcia Aparecida Jacomini - Universidade Federal de São Paulo, Brasil

\section{Conselho Editorial}

\section{Alejandro Morduchowicz}

Universidad Pedagógica, Provincia de Buenos Aires, Argentina

Fernanda Saforcada

Universidade de Buenos Aires, Argentina

Jacques Velloso

Universidade de Brasília, Brasil

João Monlevade

Senado Federal, Brasil

Jorge Abrahão de Castro

Instituto de Pesquisa Econômica Aplicada / IPEA, Brasil

Juca Gil

Universidade Federal do Rio Grande do Sul, Brasil

Lisete Regina Gomes Arelaro

Universidade de São Paulo, Brasil

Luis Carlos Sales

Universidade Federal do Piauí, Brasil

Luiz de Sousa Junior

Universidade Federal da Paraíba, Brasil

Luiz Fernandes Dourado

Universidade Federal de Goiás, Brasil

Magna França

Universidade Federal do Rio Grande do Norte, Brasil

\section{Maria Beatriz Luce}

Universidade Federal do Pampa, Brasil

Universidade Federal do Rio Grande do Sul, Brasil

Marcos Edgar Bassi

Universidade Federal de Santa Catarina, Brasil

\author{
Maria Dilnéia Espíndola Fernandes \\ Universidade Federal de Mato Grosso do Sul, Brasil \\ Nalú Farenzena \\ Universidade Federal do Rio Grande do Sul, Brasil \\ Nelson Cardoso do Amaral \\ Universidade Federal de Goiás, Brasil \\ Nicholas Davies \\ Universidade Federal Fluminense, Brasil \\ Rosana Evangelista Cruz \\ Universidade Federal do Piauí, Brasil \\ Rosana Gemaque \\ Universidade Federal do Pará, Brasil \\ Robert E. Verhine \\ Universidade Federal da Bahia, Brasil \\ Romualdo Portela de Oliveira \\ Universidade de São Paulo, Brasil \\ Theresa Adrião \\ Universidade Estadual de Campinas, Brasil \\ Tristan McCowan \\ University of London, Reino Unido \\ Vera Jacob \\ Universidade Federal do Pará, Brasil \\ Vera Peroni \\ Universidade Federal do Rio Grande do Sul, Brasil \\ Vitor Henrique Paro \\ Universidade de São Paulo, Brasil
}

\section{Equipe editorial}

Apoio ao Comitê Editorial: Patrícia Balthazar Garcia

Diagramação, Revisão de português e normalização: Edson Leonel de Oliveira

Revisão de inglês: Ananyr Porto Fajardo 